\section{Krebs-Wörterbuch für Patienten}

\section{Patienten, bei denen Krebs diagnosti- ziert wurde, haben viele Fragen. Um Ihnen zu helfen, hat die Deutsche Krebshilfe ein „Krebs-Wörterbuch“ he- rausgegeben. Die Broschüre ist kos- tenlos und auch im Internet verfüg- bar.}

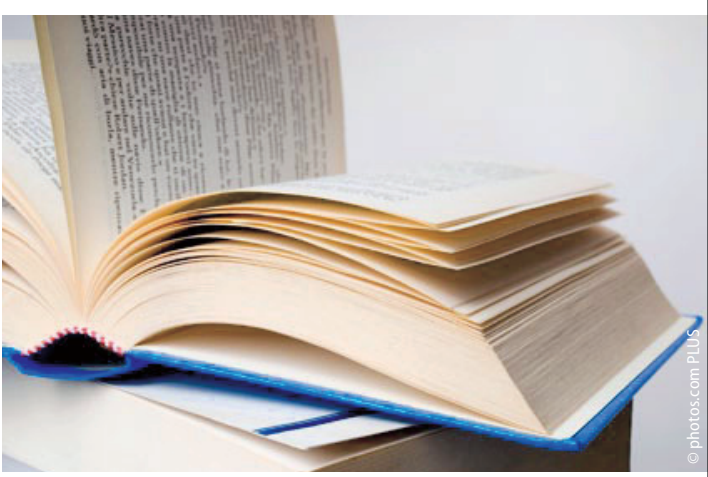

Nach der Diagnose Krebs sind Betroffene oft unsicher, haben Angst vor der Zukunft und wissen nicht, welche Therapie die richtige für sie ist. Hinzu kommt, dass viele behandelnde Ärzte Fachausdrücke benutzen, die für den medizinischen Laien nur schwer verständlich sind. In dieser Situation soll die neue Broschüre helfen. Das Wörterbuch erläutert die wichtigsten Fachbegriffe der Krebsmedizin laienverständlich, ein Abkürzungsverzeichnis enthält zudem gängige Abkürzungen. Fachbegriffe aus der Onkologie wie z.B. „Adenom“, „Ösophaguskarzinom" aber auch allgemeine medizinische Fachbegriffe wie z.B.„Biopsie“ oder "palliativ“ werden kurz erläutert. Die gemeinnützige Organisation gibt zahlreiche Broschüren heraus, in denen Diagnose, Therapie und Nachsorge der häufigsten Krebskrankheiten neutral und unabhängig erklärt werden. Alle Informationsmaterialien können unter der Telefonnummer 0228/729900 sowie auf der Internetseite www.krebshilfe.de bestellt oder heruntergeladen werden.

Quelle: Deutsche Krebshilfe
Das Mediastinum wird für die Beurteilung in 3 Kompartimente eingeteilt (- Abb. 1):

- vorderes Mediastinum zwischen Sternum und Gefäßen, einschließlich des normal gelegenen Thymus;

- mittleres Mediastinum mit Trachea, Herz, Aortenbogen, Venen, Lymphknoten, Ösophagus, Nerven;

- hinteres Mediastinum dorsal der Wirbelkörpervorderkante mit Aorta descendens, V. azygos und Nerven.

In den einzelnen Kompartimenten treten bestimmte Tumoren gehäuft auf (- Tab. 2). Gleichzeitig zeigen sie den Weg für eine aussagekräftige Biopsie auf.

\section{Wege zu einer aussagekräfti- gen Gewebeprobe}

Gut abgegrenzte Tumoren $<5 \mathrm{~cm}$ sowie zystische Raumforderungen in der Thymusloge (typischerweise Thymome vom Typ A oder AB oder Thymuszysten), peritracheal (bronchogene Zysten) oder im hinteren Mediastinum (neurogene Tumoren) ohne lokale klinische Symptomatik können primär minimalinvasiv, d. h. videothorakoskopisch reseziert werden, wenn eine komplette Resektion des Prozesses unter Vermeidung einer Tumorruptur mit an Sicherheit grenzender Wahrscheinlichkeit präoperativ möglich erscheint. Auf diese Weise werden Diagnose und Therapie bei den meist gutartigen Tumoren in einem Schritt realisiert.

\section{» Gut abgegrenzte Tumoren $<5 \mathrm{~cm}$ und zystische Raumfor- derungen lassen sich häufig minimalinvasiv resezieren «}

In allen anderen Fällen sind die breite Palette an malignen Tumoren und die unterschiedlichen Therapiekonzepte zu berücksichtigen. Im Prinzip besteht für jedes Kompartiment die Option der bildgestützen Stanzbiopsie oder der endoskopischen offenen Biopsie. Im ersten Fall ist die Biopsie relativ klein. Dies

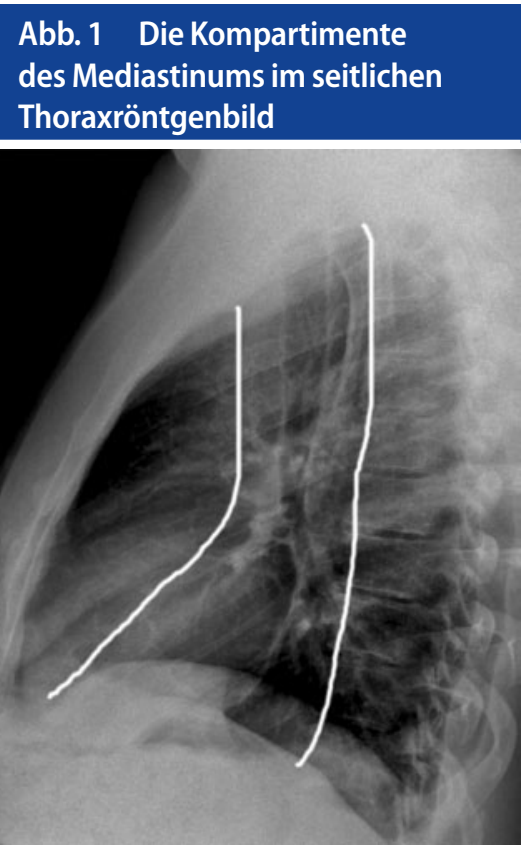

Abb. 2 Nebenschilddrüsenadenom im vorderen Mediastinum (Tc-99m-Sestamibi-Szintigraphie)

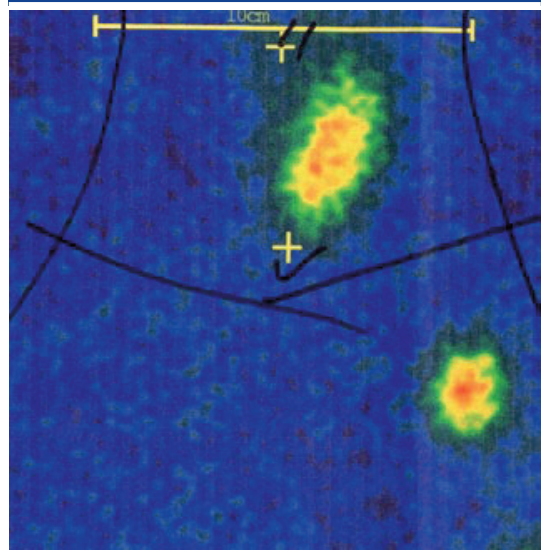

kann im Falle von aufwendigen pathologischen Untersuchungen mit Immunhistologie und Molekularpathologie gelegentlich zu Problemen bei der Einordnung der Tumoren führen und eine erneute (meist offene) Biopsie erforderlich machen.

Das vordere Mediastinum wird durch eine sonographisch oder radiologisch gestützte Stanzbiopsie transthorakal, durch eine anteriore Mediastinotomie (Inzision parasternal) oder durch eine vordere Videomediastinoskopie transzervikal erreicht. Das mittlere Mediastinum wird mittels endobronchialen Ultraschalls 